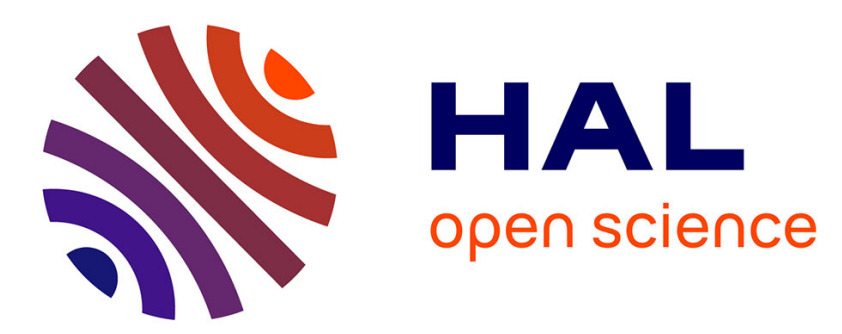

\title{
Stability criteria for yet another class of multidimensional distributed systems
}

Leonidas Georgiadis, Wojciec Szpankowski

\section{To cite this version:}

Leonidas Georgiadis, Wojciec Szpankowski. Stability criteria for yet another class of multidimensional distributed systems. [Research Report] RR-1996, INRIA. 1993. inria-00074676

\section{HAL Id: inria-00074676 \\ https://hal.inria.fr/inria-00074676}

Submitted on 24 May 2006

HAL is a multi-disciplinary open access archive for the deposit and dissemination of scientific research documents, whether they are published or not. The documents may come from teaching and research institutions in France or abroad, or from public or private research centers.
L'archive ouverte pluridisciplinaire HAL, est destinée au dépôt et à la diffusion de documents scientifiques de niveau recherche, publiés ou non, émanant des établissements d'enseignement et de recherche français ou étrangers, des laboratoires publics ou privés. 


\section{Stability Criteria}

\section{for Yet Another Class \\ of Multidimensional \\ Distributed Systems}

Lconidas GEORGIADIS

Wojciech SZPANKOWSKI

$\mathrm{N}^{\circ} 1996$

Juillet 1993

FROGRAMME 2

Calcul symbolique,

programmation

et génie logiciel

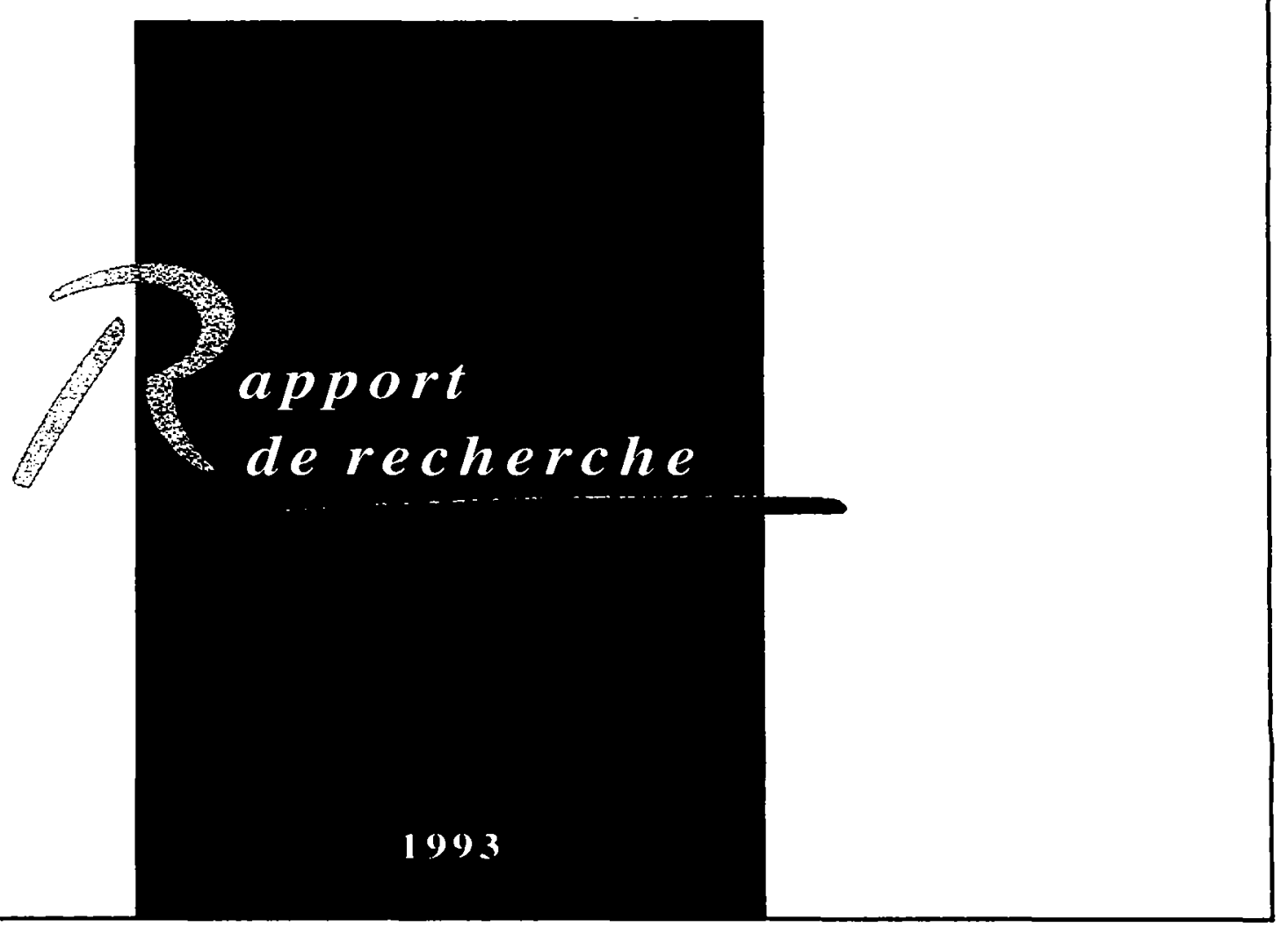




\title{
STABILITY CRITERIA FOR YET ANOTHER CLASS OF MULTIDIMENSIONAL DISTRIBUTED SYSTEMS
}

\author{
September 1991
}

Reviserl: .June 30, 1993

Leonidas Georgiadis

IBM T. J. Watson Research Center

P.O. Box 704

Yorktown Heights, NY 10598

U.S.A.

leonid@watson.ibm.com

\author{
Wojciech Szpankowski* \\ Department of Computer Science \\ Purdue University \\ W. Lafayette, IN 47907 \\ U.S.A. \\ szpankow@marganx.inria.fr
}

\begin{abstract}
We present sufficient and necessary conditions for stability of token passing rings with time-limited discipline and more generally, a class of monotonic and contractive service disciplines. In general, estalslishing stability for multidimensional distributed systems is a difficult problem. The standard Lyapunov test function method often fails when applied to such systems (e.g., token passing rings, ALOHA-type systems, rings with spatial reuse, couple-processors system, etc.). In our recent work on this topic we establish a useful alternative approach that turus ont to be successful for deriving stability conditions for several distributed systems. In the current paper, we show that our approach can be extended so that it can be applied to the system under consideration.
\end{abstract}

\section{CRITERES DE STABILITE POUR UNE NOUVELLE CLASS DE SYSTEMES DISTRIBUE MULTIDIMENSIONNELS}

\section{Résumé}

Nous prẹsentons des conditions nécessaires et suffisantes pour la stalbilité d'anneaux à jeton avec discipline de service sous contraintes temporelles. En général, la stabilité de systèmes multidimensionuels est un problème difficile. Les tests à la Lyapunov y sont souvent voués à l'echec (annenu à jeton, systèmes de type ALOHA, annean avec réutilisation spatiale, couplage de processenurs, e.tc.). Dans un de nos récents travaux sur ce sujet nous établissons une méthorle alternative qui s'avère efficace pour établir la stabilité de nombreux systèmes. Dans ce papier, nous montrons qute notre approche pent être étendue à une classe plus large de systèmes.

*This research was supported in part by the NSF grants NC:R-9206315, C(:R-9201078, aud INT-8912631, by AFOSR grant $9(1-0107$, and in part hy NATO (ollaborative: (irant 01057/89. This paper was revised while: the author was visiting INRIA, Rocenteucourt., France, and he wisles to thank INRIA (projects ALGO, MEVAL and REFLECS) for a generons support. 


\section{INTRODUCTION}

Distributed multiquene syst.ens which share a single scarce resource (i.e., server) such as a communication channel or a processor, have received a considerable amount of attention in the recent literature. Of special interest are token passing rings (cf. [5], [16], [17]) due to a number of reasons. For example, it is known that obtaining the distribution of the number of messages quened in each station is a formidable open problem, as is the problem of obtaining the waiting time distribution. The stalvility condition for the token passing ring was heuristically predicted by Kuehn [5] in 1979, and then reproduced with some minor changes in many other papers (cf. [4]). Recently, in our paper [3] we have established rigorously stability conditions for the P-linited token passing rings, and indicated that the technique can be extended to other disciplines We complete this study in the current. paper. Specifically, we apply our methodology to derive stability criteria for time-limited token passing rings introduced recerttly by Leung and Eisenberg [(i], [7] (cf. Proposition 1). In such a system each station transmits messages for at most an amount of time $\tau$. If the transmission time exceeds $\tau$, the station completes the transmission of the message in progress and sends the token to the next station (the so called nonpreenptive time limited discipline). While we sturly this system as an important application, the technique can be applied almost without modification to a class of monotonic and contractive policies (cf Proposition 2).

Our approach to the stability of token passing rings follows the idea discussed in our paper [3], and differs significantly from the standard methodology of the Lyapunov test. function (cf. [12], [18], [14]). (For other than test function approaches see also [2], [11] $[14,15]$.) It resembles the general idea of Malyshev's faccs and inducrd Markon chains [10]. Our method is based on a simple irlea of stochastic dominance technique, and application of Loynes [9] stability criteria for an isolated quene. We note that this approach is not restricted to token passing rings, and stalbility of several other distributed systems can be assessed by this methorlology (cf. $[14,15]$ ).

We now summarize our main resnlts. We shall analyze the token passing ring with Poisson arrivals with parameter $\lambda_{i}$ for the $i$ th station, general distriluntion of service times $\left\{S_{i}^{k}\right\}_{k=1}^{\infty}$ and switchover times $\left\{U_{i}^{k}\right\}_{k=1}^{\infty}$. We consicler a gated version of $\tau$-limited token ring. Define $\tilde{L}_{i}=\min \left\{k: \sum_{j=1}^{k} S_{i}^{j} \geq \tau_{i}\right\}$ and $\ell_{i}=E \tilde{L}_{i}$. (learly, $\ell_{i}$ is the average of the maximum number of customess served during $\tau_{i}$.

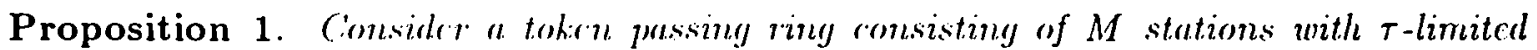
scrvice scherdule for the ith station, and Porisson arrinals. Then the system is stable if and 
only if $\sum_{j=1}^{M} p_{j}<1$ anul

$$
\lambda_{j}<\frac{\rho_{j}}{u_{0}}\left(1-\rho_{0}\right) \text { for all } j \in \mathcal{M}=\{1, \ldots M\},
$$

where $u_{0}=\sum_{i=1}^{M} E U_{i}$ is the: anverwge: totul switrhonwr time, anul $\rho_{0}=\sum_{i=1}^{M} \rho_{i}$ with $\rho_{i}=\lambda_{i} s_{i}$ and $s_{i}=E S_{i}$ being the average scrvise: time: at the ith station.

It will be seen that the method of the proof can he applied virtually unchanged to the following class of monotonic and contractive policies. We assume that the number of customers served from cuene $i$ when there are "n quened messages at the instant of token arrival to quene $i$ is $f_{i}(n, \mathrm{X})$, where $\mathrm{X}$ is a possibly random quantity that depends on the policy. We assume that $f_{i}(\cdot, \mathrm{X})$ is a nondcrcrcasing function of the number of customers in the $i$ th quene. In addition, the following relation holds

$$
f_{i}\left(n_{1}, \mathbf{X}\right)-f_{i}\left(n_{2}, \mathbf{X}\right) \leq n_{1}-n_{2} \quad \text { if } \quad n_{1}>n_{2} \text {. }
$$

At the $k$ th token arrival to quene $i$ the cuantity $\mathbf{X}$ takes the value $\mathbf{A}_{k}$. The random variables $\left\{\mathbf{A}_{k}\right\}_{k=1}^{\infty}$ are i.i.d, are independent of the past arrival times, service times and switchover times. Note that $f_{i}\left(n, \mathbf{A}_{k}\right)$ tends to a randon variallele, $f_{i}^{*}\left(\mathbf{A}_{k}\right)$, when $n-\infty$. We assume that $f_{i}^{*}\left(\mathbf{A}_{k}\right)$ is finite and

$$
\ell_{i} \stackrel{\text { leff }}{=} E f_{i}^{*}\left(\mathbf{A}_{k}\right)<\infty
$$

We will see in the next section that the time-limited token ring policy falls within the class just described. Other special cases are the f-linited gated discipline and Bernoulli gated disciplines. In the last descipline, $f_{i}(n, X)=\min \{n, X\}$, where $X$ is a geometrically distributed random variable. The following result follows directly from the arguments that will be presented in this paper.

Proposition 2. Considrr a tokkrn passing ring that cmployss a monotonic: and contractive service discipline described above. Then, the syste:m is stable: if and only if

$$
\lambda_{i}<\frac{P_{i}}{u_{11}}\left(1-p_{10}\right) \quad \text { for all } \quad i \in \mathcal{M}
$$

uhere $\rho_{0}<1$.

One policy that does not fall in the previously described category is the promptive $\tau$ limited token ring. In such as system the token interrupts his service inmediately after the time limit $\tau$ expires and continnes servicing the same customer in the next romul. While the formal argunents of onr methodology can be applied for this system, there are technical difficulties that need to be overcome for a completes proof. We helieve that these technical 
arguments can be provided, hut we do not have a complete proof yet, and therefore we express the following conjectinte.

Conjecture. The premptine $\tau$-limitcd tokicn ring is stable if and omly if

$$
\lambda_{j}<\frac{\tau_{i}}{s_{j} u_{10}}\left(1-\rho_{0}\right) \text { for all } j \in \mathcal{M}=\{1, \ldots M\},
$$

and $\rho_{0}<1$.

The paper is organized as follows. In the next section we present some prelininary results In particular, we find Markovian representations of the system, prove a crucial stochastic. dominance relationship, and establish some Wald's type formulas. Finally, in Section 3 we present our main construction that leads to the proof of the Proposition 1. Since the proof of Proposition 2 is along the lines of the proof of Proposition 1, we shall concentrate only on establishing Proposition 1, leaving the details of the proof of Proposition 2 to the reader.

\section{PRELIMINARY RESULTS}

In this section we present. several results that are required to establish our main finding regarding the stability of the token passing ring. These results are of their own interests, and can be used to obtain some estimates for the performance evaluation of the system. In the sequel, we list our main assunptions, describe the Markovian character of an imbedcled queneing process, show two simple Wald's type irlentities, and finally establish a stochastic dominance relationship.

We start with a precise definition of onr stochastic model. We shall adopt the following assumptions.

(A1) There are $M$ stations (cuneues) on a. loop, each having infinite capacity buffer.

(A2) Maximnm time customers are served during the token visit at a quene is linited to $\tau_{i}<\infty$ units of time. (Only customers that are present at the instant of token arrival can be served. Moreover, we lave nonpreemptive discipline, that. is, the customer that is in the server when the time limit $\tau_{i}$ is reached, is served to completion lefore the token moves to the next quene.

(A3) Arrival proces $A_{i}^{t}, t \in[0, \infty)$ to the $i$ th quene is a Poisson process with parameter $\lambda_{i}>0$. Here, $A_{i}^{t}$ is the number of arrivals at quene $i$ up to time t. The arrival process at a cuene is indepenendent of the arrival processes to other quenes. 
(A4) Service time process $\left\{S_{i}^{k}\right\}_{k=1}^{\infty}$ at. queme $i$ is i.i.cl. with $s_{i}=E S_{i}^{1}>0$. The service time process at a quene is independent of the arrival processes at all quenes and independent of the service timue processes at other quenes.

(A5) The switchover times letwen $i$ and $i+1 \bmod M$ quene, $\left\{U_{i}^{k}\right\}_{k=1}^{\infty}$, are i.i.d., independent of the switchover tines $\left\{U_{j}^{k}\right\}_{k=1}^{\infty}$ for $j \neq i$, and independent of the arrival and the service time processes. The average total switchover time is defined as $u_{0}=\sum_{i=1}^{M} E U_{i}^{1}$. To avoid unnecessary complications we assume that $P\left(U_{i}^{n}>0\right)=1, i=1, \cdots, M$.

Now we are ready to present a Markovian description of the system. We need a little bit of notation. By (A1), the token visits stations in a cyclic order. Let $n$ clenote the $n$th visit of the token to any queue. Then, $k_{n}=\lfloor(n-1) / M\rfloor+1$ denotes the cycle number in which the $n$th visits occurs (we start comuting cycles from one and assume that the token starts from queue 1). Note that the (luene visited at the $n$th visit is just $J_{n}=n-M\left(k_{n}-1\right)$. Let also $\left\{T_{n}\right\}_{n=1}^{\infty}$ he the time instant of the $n$th visit of the token to any quene. Define an $M$-dimensional process $\tilde{\mathrm{N}}^{n}=\left(\tilde{N}_{1}^{n}, \ldots, \tilde{N}_{M}^{n}\right), \quad n=1,2, \ldots$, where $\tilde{N}_{i}^{n}$ as the number of customers in quene $i$ at time $T_{n}$. In addition, by $\mathcal{N}_{i}^{n}$ we mean the total number of customers served from quene $i$ up to time $T_{n}$. Theorem 1 below shows that $\tilde{\mathrm{N}}^{n}$ is a Markov chain. Since its proof is along the lines of our Theorem 1 from [3], we omit it here.

Theorem 1. The proccsss $\tilde{\mathrm{N}}^{\prime}$ is a uonhonnoygrnoms Markon chain.

Remark: It should be noted that the assumption that the service discipline is nonpreemptive (see assumption $(A 2)$ ) is crucial for Theorem 1 to hold. Assume, for example, that preemptions were allowed so that a server could interrupt the service of the customer as soon as the limit $\tau$ was reached. Upon the next arrival of the token to the quene, the server could either complete the remaining service time or restart the service time of the interrupted customer. In both cases, the number of customers in quene $i$ at time $T_{n}$ will depend in general on the service time $S_{i}^{\mathcal{N}_{i}^{\prime \prime}+1}$ and the process of quene lengths will not be Markov.

There are other Markovian descriptions of the system. For example, define $N_{j}^{n}(i)$ to be the number of customers at quene $j$ when the token visits quene $i$ for the $n$th time. Then, the process $\mathrm{N}^{n}(i)=\left(N_{1}^{n}(i), \ldots, N_{M}^{n}(i)\right)$ can be deduced from $\tilde{\mathrm{N}}^{n}$ since $\mathrm{N}^{n}(i)=\tilde{\mathrm{N}}^{(n-1) M+i}$ and therefore it is a Markov chain. It is not difficult to verify also that,

Corollary 2. The proress $\mathrm{N}^{\prime \prime}(i)$ of the qurue lengthes registered by the token when it visits

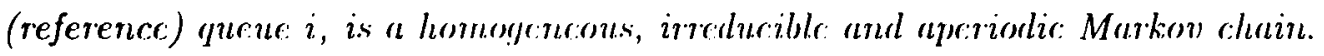


The fact that under assumption (A2) the service times of the customers at quene $i$ at instant $T_{n}$ are i.i.d. independent of the quene size at time $T_{n}$, permits us to consider a new morlel of the system which is stochastically equivalent to the original one and has the arlvantage that uncler this new nodel, many of the arguments that follow become simpler. Specifically, in the new system assimuptions (A1)-(A3) and (A5) are the same, while assumption (A4) is replacerl with

(A4') Service times are assigned to the customers at quene $i$ upon beginning of service as follows. We consirler a doubly infinite sequence of i.i.cl random variables $\left\{S_{i}^{n, k}\right\}_{n, k=1}^{\infty}$ with $s_{i}=E S_{i}^{n, k}>0$. The customers that are served during the $u$ th arrival of the token to quene $i$ are assigned the service times $S_{i}^{n, 1}, S_{i}^{n, 2}, \ldots$. The serpuence $\left\{S_{i}^{n, k}\right\}_{n, k=1}^{\infty}$ is independent of the sequence $\left\{S_{, j}^{\prime \prime, k}\right\}_{n, k=1}^{\infty}$ for $i \neq j$, and independent of the interarrival processes to the quenes.

Next, we need some relationships between the average number of customers served per token visit $L_{i}^{n}$ and the average cycle time $C_{i}^{\prime \prime}$. The former quantity is defined as follows. Let $\widetilde{L}_{i}^{n}=\min \left\{k: \sum_{j=1}^{k} S_{i}^{n, j} \geq \tau_{i}\right\}$. Then $L_{i}^{n}=\min \left\{\tilde{L}_{i}^{n}, N_{i}^{n}\right\}$. The latter quantity is the length of time between the $n$th and $n+1$ st visits of the token to the reference queue $i$. By $E L_{i}$ and $E C$ we denote the liniting averages of $L_{i}^{n}$ and $C_{i}^{\prime \prime}$. It turns out that the relations holding for the l-limited case, continue to hold under the more general policies we consider here. Specifically, we have the following result.

Theorem 3. Let the Murkov chain $\mathrm{N}^{\prime \prime}(i)$ be positive rcurrent (eryodic) for some $i \in \mathcal{M}$. Then, $\mathrm{N}^{n}(j)$ is ergodic for all $j \in \mathcal{M}$, and $m_{0}=\sum_{j=1}^{M} \rho_{j}<1$. In addition,

$$
\begin{aligned}
E L_{j} & =\lambda_{j} E C:, \quad j \in \mathcal{M} \\
E C & =\frac{u_{0}}{1-\sum_{j=1}^{M} \mu_{j}},
\end{aligned}
$$

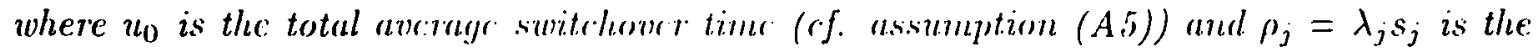
utilization coefficicnt for the ith quene.

Proof. We need only minor modifications compared to the proof of our Theorem 3 in [3]. We present a brief sketch of the proof. Without loss of generality, let $i=1$. Define

$$
K^{-n+1}=\min \left\{m>K^{n}: N^{\prime \prime \prime}(1)=0\right\}
$$

and $R^{n}=K^{n+1}-K^{n}$. We also write $R=R^{1}$. Due to the ergodicity of $\mathrm{N}^{n}(1)$ we have $E R<\infty$. Observe that for $j \in \mathcal{M}$, the process $\mathrm{N}^{n}(j)$ is regenerative with respect to $R^{n}$. 
Therefore (cf. [1]), alunost. surely

$$
\lim _{n \rightarrow \infty} \frac{\sum_{k=1}^{n} L_{j}^{k}}{n}=\frac{E\left(\sum_{k=1}^{k i} L_{j}^{k}\right)}{E R}=E L_{j} \quad, \lim _{n \rightarrow \infty} \frac{\sum_{k=1}^{n} C_{1}^{k}}{n}=\frac{E\left(\sum_{k=1}^{k} C_{i}^{k}\right)}{E R}=E C_{1} .
$$

Let now $\tilde{L}_{i}^{n}=\min \left\{k: \sum_{j=1}^{k} s_{i}^{i n, j} \geq r_{i}\right\}$. Clearly, $\tilde{L}_{i}^{n}, n=1,2, \cdots$ are i.i.d. random variables and since $s_{1}>0$, it is well known from renewal theory that $E \tilde{L}_{1}^{n}=\ell_{1}<\propto$. Observe also that the event $\{R \leq k\}$ is indegendent of the sednence $\tilde{L}_{1}^{n}, n>k$ and therefore, $E\left(\sum_{k=1}^{R} \tilde{L}_{1}^{k}\right)=l_{1} E K<\infty$. Note that in the interval $\left[0, \sum_{k=1}^{R} C_{1}^{k}\right)$ all the arriving customers from all rucues must be scrocrl. If $\mathcal{A}_{j}$ is the number of arrivals to quene $j$ in the interval $\left[0, \sum_{k=1}^{R} C_{1}^{k}\right)$, then $E \mathcal{A}_{j}=E\left(\sum_{k=1}^{R} L_{j}^{k}\right)$, and due to the Poisson assumption (A3) we also have

$$
\lambda_{j} E\left(\sum_{k=1}^{F}\left(\begin{array}{l}
k \\
i
\end{array}\right)=E\left(\sum_{k=1}^{F_{i}} L_{i}^{k}\right) \leq E\left(\sum_{k=1}^{F} \tilde{L}_{1}^{k}\right)<\infty\right.
$$

The above and (3) lead to $E L_{j}=\lambda_{j} E C_{1}$, which completes the proof of (1). The proof of (2) is similar.

The next result is one of the key elements of our stability analysis. We first give a brief overview of our approach, avoiding techinical details. In the process of estimating stability we need to build several dominant systems of the original token passing ring. For example, when we study stalility of an isolated station, say the $j$ th one, we partition all other stations into a class $\mathcal{S}$ of nonprersistent queues and a class $\mathcal{L}$ of persistcnt quenes (saturated queues). A nonpersistent quene serves customers in the normal way as in the original token passing ring. A persistent quene, however, always sends the maximum allowable number of customers, that is, $\widetilde{L}_{i}$ for $i \in \mathcal{U}$, ly sending if necessary dummy customers. A question is whether such a new system dominates the original token passing ring in some sense. If the answer is yes, then by proving stalbility of the dominant system we establish stability of the original token passing ring. In passing, we note that persistent and noupersistent quenes are equivalent to "faces" and "induced Markov chiains" in the Malyshev terminology ([10]).

We state the next result in a general form, since it is needed to prove Proposition 2. Specifically, in the terminology of $[\$]$, we consider the class of monotonic, contractive policies. This amounts to replacing assumption (A2) with the following more general one.

(A2') Let $\mathbf{A}$ denote a secpuence of real numbers $\left\{a_{1}, a_{2}, \cdots\right\}$. Let $f_{i}(m, \mathbf{A})$ be the number of customers served from quene $i$ when there are $m$ quened messages at the instant of the $n$th token arrival at quene $i$ and $\left\{S_{i}^{n, 1}, S_{i}^{n, 2}, \cdots\right\}=\mathbf{A}$. We assume that for fixed 
$\mathbf{A}, f_{i}(m, \mathbf{A})$ is a nondecreasing function of $m$. In addition, for a fixed $\mathbf{A}$, the following relation holds

$$
f_{i}\left(m_{1}, \mathbf{A}\right)-f_{i}\left(m_{2}, \mathbf{A}\right) \leq m_{1}-m_{2} \quad \text { if } \quad m_{1}>m_{l_{2}} .
$$

For the case of $\tau$-limited policy we liave that

$$
f_{i}\left(m,\left\{S_{i}^{n, 1}, S_{i}^{n, 2}, \cdots\right\}\right)=\min \left\{m, \tilde{L}_{i}^{n}\right\}
$$

Now we are ready to formulate om result. Consider two token passing rings, say $\theta$ and $\Theta$. Both satisfy assumptions (A1)-(A5) with (A2) replaced by the weaker assumption (A2'). The system $\theta$ represents our original token passing ring. The system $\Theta$ differs only in the switchover times, namely, we assmme that the switchover time for $\Theta$ is replaced by $\left\{\Delta_{i}^{k}+U_{i}^{k}\right\}_{k=1}^{\infty}$ for $i=1, \cdots, M$. We assume that for every $i \in \mathcal{M}$ and every $k \geq 0$ we have $\Delta_{i}^{k} \geq 0$. We make the following assumption for the process $\Delta_{i}^{k}$.

(A6) The random variable $\Delta_{i}^{k}$ is independent of the service times, switchover times and the Poisson increments of the arrival processes to all stations after time $T_{M(k-1)+(i+1)}-U_{i}^{k}$ (see Fig.1).

Theorem 4. Let $\tilde{\mathrm{N}}^{\prime \prime}(\theta)$ and $\tilde{\mathrm{N}}^{\prime \prime}(\Theta)$ drnote the que uc: lengths in both systems. Then, under the above assumptions.s, and undrer the condition that the token starts from the same queue, say queue number one:, and with the: samu: number of initiol customers in both systems, the following holds

$$
\tilde{\mathrm{N}}^{u}(\theta) \leq_{s t} \tilde{\mathrm{N}}^{u}(\Theta)
$$

where $\leq_{s t}$ mons stoclustically suuallcr.

Proof. The proof is along the lines of Theorem 4 our paper [3]. For completeness in the presentation, we provicle some details. To avoid cumbersome notation we present the proof only for $M=2$ nsers.

We define some new variables. For a system $\theta$ let, $T_{n}^{\theta}$ and $D_{n}^{\theta}$ denote the instances of the $n$th visit and the $n$th token departure from any duene respectively. As before, $J_{n}^{\theta}$ denotes the quene number visiterl at the $u$ th visit of the token. Finally, $L_{i}^{n}(\theta)$ as before denotes the number of customers served from duente $i$ at the $n$th visit of the token. Clearly, for our two station system $L_{1}^{\prime \prime}(\theta)=0$ for $"$ even, and $L_{2}^{\prime \prime}(\theta)=0$ for $n$ odd. In a similar manner we define respective (quantitities in the $\theta$ system. 


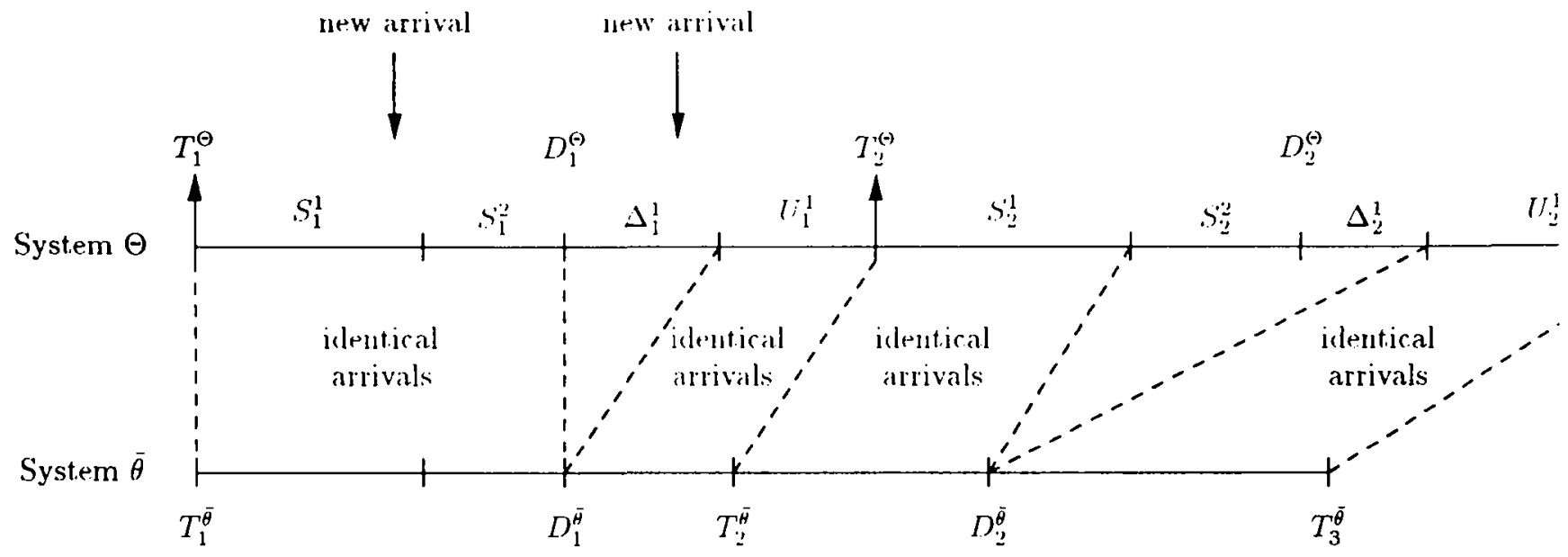

Figure 1: Illustration to the poof of Theorem 4

We will construct from the system $(-)$ a tohen passing ring $\bar{\theta}$, which is stochastically equivalent to the system $\theta$ and for which we have that

$$
\tilde{\mathbf{N}}^{\prime \prime}(\bar{\theta}) \leq \overline{\mathbf{N}}^{\prime \prime}(\Theta)
$$

Figure 1 should help to muderstand our construction. Assinue $\tilde{N}_{i}^{1}(\bar{\theta})=\tilde{N}_{i}^{1}(\Theta)$ for $i=1,2$. The service times in system $\bar{A}$ are assigned from the salne secpuences $S_{i}^{n, k}$ as in $\Theta$ (according to assumption (A4').) Also, the sune functions $f_{i}(m, \mathbf{A}), i=1,2$ are used in both systems. Therefore, the decision to switch to guene 2 will occur at the same time, namely $D_{1}^{\bar{\theta}}=D_{1}^{\Theta}$. The switchover time for $\bar{\theta}$ beromes now $U_{1}^{1}$, and of course $T_{2}^{\bar{\theta}} \leq T_{2}^{\Theta}$ since $\Delta_{1}^{1} \geq 0$ (see Fig. 1).

The arrivals in the system $\dot{\theta}$ in $\left[D_{1}^{\bar{\theta}}, T_{2}^{\bar{\theta}}\right)$ are: mow assunurd to be idcntical to the arrivals in $\left[D_{1}^{\Theta}+\Delta_{1}^{1}, T_{2}^{\Theta}\right)$ in $\Theta$ system. Therefore clearly $\tilde{N}_{i}^{2}(\bar{\theta}) \leq \tilde{N}_{i}^{2}(\Theta)$ for $i=1,2$. The arrivals to system $\bar{\theta}$ in $\left[T_{2}^{\bar{\theta}}, T_{2}^{\bar{\theta}}+S_{2}^{1}+\cdots+S_{2}^{L_{2}^{1}(\dot{\theta})}\right)$ are taken to be iclentical to the arrivals in $\left[T_{2}^{\Theta}, T_{2}^{\Theta}+S_{2}^{1}+\cdots+S_{2}^{L_{2}^{1}(\bar{\theta})}\right)$. Note that this can be done since by $\left(\mathrm{A} 2^{\prime}\right) L_{2}^{1}(\bar{\theta}) \leq L_{2}^{1}(\Theta)$. Observe also that $T_{2}^{\bar{\theta}}+S_{2}^{1}+\cdots+S_{2}^{L_{2}^{L}(\bar{\theta})}=D_{2}^{\theta}$ (Fig. 1).

To complete the description of the system $\ddot{\theta}$ we have to sperify the arrivals in $\left[D_{2}^{\bar{\theta}}, D_{2}^{\bar{\theta}}+\right.$ $\left.U_{2}^{1}\right)$. These are taken to be exactly the arrivals in $\left[D_{2}^{(-)}+\Delta_{2}^{1}, D_{2}^{(-)}+\Delta_{2}^{1}+U_{2}^{1}\right)$ in the clominant system $\Theta$ (see Fig. 1). Note from the construction that

$$
\tilde{N}_{1}^{3}(\bar{\theta})=\tilde{N}_{1}^{2}(\bar{\theta})+A_{\left[T_{2}^{*}, T_{:}^{(\dot{\theta}]}\right]} \leq \tilde{N}_{1}^{2}(\Theta)+A_{\left[T_{2}^{(-)}, T_{3}^{(-)}\right]}=\tilde{N}_{1}^{3}(\Theta)
$$


and also i,y (5)

$$
\tilde{N}_{2}^{3}(\bar{\theta})=\tilde{N}_{2}^{2}(\bar{\theta})-L_{2}^{2}(\bar{\theta}) \leq \tilde{N}_{2}^{2}(\Theta)-L_{2}^{2}(\Theta)=\tilde{N}_{2}^{3}(\Theta)
$$

We can now repeat exactly the same procedure to construct $\bar{\theta}$ in the interval $\left(T_{n}^{\bar{\theta}}, T_{n+1}^{\bar{\theta}}\right), n \geq$ 3 , in the same manner as it was comstructed in the interval $\left[T_{2}, T_{3}\right)$. By construction the service times and switchover times of system $\bar{\theta}$ are identically distriluted to the corresponding variables of system $\theta$ and are independent of the interarrival process. In addition, assumption $(A(i)$ and the fact t.lat. the servicing policy is nonanticipative assures that the times $T_{n+1}^{\Theta}-U_{J_{n}}^{k_{n}}$ are stopping times for the Poisson arrival processes to all stations. The independence of the increments of the Poisson process implies now, that the constructed interarrival process in system $\bar{\theta}$ is Poisson with rate $\lambda_{i}$ for quene $i$. Moreover, by construction (7) holds. Since $\bar{\theta}$ is storlastically erpivalent to $\theta$, we have that the distribution of $\tilde{\mathrm{N}}^{n}(\theta)$ is identical to the distribution of $\tilde{\mathrm{N}}^{\prime \prime}(\bar{\theta})$. This completes the proof of Theorem 4.

\section{MAIN RESULTS}

Our approach in this section is baserl on three simple olservations. At first, we note that a multidimensional process is stalle if and only if its components are stable [14]. More precisely, if $\mathrm{N}^{n}=\left(N_{1}^{n}, \ldots, N_{M}^{n}\right)$ is a stoclastic process - not necessary a Markov chain then say the process is stable if the clistribution of $\mathrm{N}^{\prime \prime}$ ass $n-\infty$ caists and the distribution is honest (cf. $[9,14,19]$ ).

Sccondly, to obtain stability conditions for a single isolated station in the token passing ring, we apply the techuique of Loynes [?] who proverl that a single (i|Ci|1 quene is stable if the input rate is smaller than the average service time provided that service times and interarrival times are jointly stationary and ergodic. To verify a technical stationarity condition in Loynes' criteria we apply the stochastic clominance result of Theorem 4. More precisely, we partition the set of quenes, $\mathcal{M}$, into a set. $\mathcal{S}$ of nonpersistent quenes and into a. set $\mathcal{U}$ of persistent cquenes as was described in Section 2 . By Theorem 4 the new system stochastically clominates the original one, and by proving stability of it, we clearly establish stability conditions for the original toke+n passing ring. We nse induction to establish stability conditions for the nonpersistent quenes in the new system, while the stability condition for a persistent cluene is established by using Loynes criteria.

To fulfill the ahove plan, we stant by showing a result that will be useful in proving the condition for a persistent quene in the dominatnt system. More formally, as in Section 2 we consider a doubly infinite serpuence of i.i.d random varialoles $\left\{S^{n, k}\right\}_{n, k=1}^{\infty}$ and recall the definition $\tilde{L}^{n}=\min \left\{k: \sum_{j=1}^{k}, S^{\prime \prime \prime} \geq \tau\right\}, 0 \leq \tau<\infty$. We consider further a quene 
with vacations such that upon the nth arrival of the server to the quene, $\tilde{L}^{n}$ custoners (dummy if necessary) are served and then the server goes for a vacation. The service times of the customers served during the mth visit of the server are the random variables $S^{n, k}, k=1, \cdots, \tilde{L}^{n}$. Let $\left\{C^{\prime \prime \prime}\right\}_{n=1}^{\infty}$ be the process of cycle times (time intervals between successive visits to the (quene). It is assumed that the processes $\left\{C^{\prime \prime}, \tilde{L}^{n}\right\}_{n=1}^{\infty}$ are jointly stationary and rygotio (no independence is recpuired). The amival process $A^{t}$ to this quene is a Poisson process with parameter $\lambda$, inclependent of the processes $\left\{C^{\prime \prime}, \tilde{L}^{n}\right\}_{n=1}^{\infty}$. Let $N^{n}$ represent the quene length at the beginning of the $n$th cycle. By $X^{n}$ we denote the number of customers arrived during the wth cycle. Since $A^{t}$ is Poisson and independent of the processes $\left\{C^{n}, \tilde{L}^{n}\right\}_{n=1}^{\infty}$, the processes $\left\{X^{n}, L^{n}\right\}_{n=1}^{\infty}$ are jointly stationary and ergodic, and $E X=\lambda E C$ : where $E C^{\prime}=E C^{\prime}{ }^{\prime}$. (') learly, the process of quene lengths at the instants of the visits of the server to the quene satisfies the following recurrence

$$
N^{n+1}=\max \left\{N^{n}+X^{n}-\tilde{L}^{n}, X^{n}\right\}, \quad n=1,2, \cdots
$$

Let $\ell=E \widetilde{L}^{n}$. We prove the following stalility result.

Lemma 5. Comsider the: queucing systrm just dessribed. If $\lambda E C^{\prime}<\ell$, then the queue is stable.

Proof. We apply Loynes' scheme to prove the lemuna. We may assume without loss of generality that $X^{n}, \tilde{L}^{n}$ is a two-sided stationary process, that is, it is defined for $-\infty<n<$ $\infty$. Note next, that the recursion ( 8 ) is such that the RHS of it represents a nondecreasing and left continnous (in $N^{n}$ ) function. Therefore, by Lemma 1 in Loynes [9] we conclude that there exists a stationary secpuence $\mathcal{N}^{k}$ satisfying recursion (8), such that $N^{n}$ converges in distribution to $\mathcal{N}^{1}$ provided that $N^{1}=0$. Now, we need to find out when $\mathcal{N}^{k}$ is honest. Recursion (8) is not quite the same as the one treated by Loynes, however we can use similar arguments as follows.

By telescoping the recurrence (\$) we immerliately olbtain for $n \geq 2$

$$
N^{n}=\operatorname{minx}_{1 \leq r \leq n-1}\left\{X^{r}+\sum_{k=r+1}^{n-1} \bar{X}^{k}\right\} .
$$

where $\bar{X}^{k}=X^{k}-\widetilde{L}^{k}$, provided $N^{1}=0$. Argning as in Loynes [9] we have that $\mathcal{N}^{k}$ is honest if and only if

$$
\lim _{r \rightarrow \infty}\left\{X^{-r}+\sum_{k=1}^{r} \bar{X}^{-k}\right\}<\infty .
$$


Observe now that

$$
X^{-r}+\sum_{k=1}^{r-1} \bar{X}^{-k}=r\left(\frac{\sum_{k=1}^{\prime} X^{-k}}{r}-\frac{r-1}{r} \frac{\sum_{k=1}^{r-1} \dot{l}^{-k}}{r-1}\right), \quad r=2,3, \cdots
$$

Since by the ergodicity of the secluences $X^{n}, \tilde{L}^{n}$ we have that $\lim _{r \rightarrow \infty}, \sum_{k=1}^{r} X^{-k} / r=\lambda E C$; and $\lim _{r \rightarrow \infty} \sum_{k=1}^{r} \tilde{L}^{-k} / r=\ell$, the condition $\lambda E($ : $<$ P. assures the validity of $(10)$. The assumption that $N^{1}=0$ can be removerl ass in [?].

Now we are ready to prove on main result described in Proposition 1. In the next theorem we show that the conditions of the Proposition are sufficient.

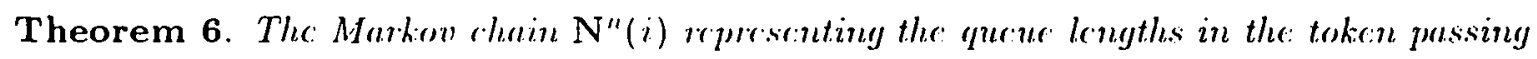
ring when it visits queur $i \in \mathcal{M}$ is cryolic if

$$
\lambda_{j}<\frac{\ell_{j}}{u_{01}}\left(1-\rho_{11}\right) \quad \text { for all } \quad j \in \mathcal{M}
$$

where $\rho_{0}=\sum_{j=1}^{M} \rho_{j}$ and $\rho_{i}=E \widetilde{L}_{i}$.

Proof. We use mathematical induction. For $M=1$ the proof is simple since it directly follows from Foster's criterion (cf. [12], [1s]).

Now we assume that the theorem is true lor $M-1$ and prove that it can be extended to the $M$ quene case. Let $(U, S), \mathcal{U} \neq \emptyset$, be a partition of the set $\mathcal{M}$ of $M$ quenes into persistent and nonpcrsistent quenes. Note that the carclinality $|S|$ of $S$ is not larger than $M-1$. Let $\overline{\mathrm{N}}^{n}(i)=\left\{\bar{N}_{1}^{n}(i), \cdots, \bar{N}_{M}^{n}(i)\right\}$ be the quene lengths when the token visits the ith quene for the $n$th time in the $(\mathcal{A}, S)$ system in which persistent quenes $\mathbb{A}$ send dummy packets as discussed above. Olserve that the modified system differs from the original token ring system only in the switchover time from a persistent. quene to the successor of that quene in the ring. Specifically, if $i \in \mathcal{H}$, then the switchover times become,

$$
\bar{U}_{i}^{k}=\Delta_{i}^{k}+U_{i}^{k}
$$

where $\Delta_{i}^{k}$ is the time neerled to service the dummy messages at norle $i$ (if any), and $\Delta_{i}^{k}$ satisfies condition ( $A(6)$ of Section 2 . Therefore, according to Theorem 4 , if $\mathrm{N}^{1}(1)=\overline{\mathrm{N}}^{1}(1)$, then

$$
\mathrm{N}^{\prime \prime}(j) \leq_{n} \overline{\mathrm{N}}^{\prime \prime}(j), \quad \text { for all } n, \quad j \in \mathcal{M}
$$

Note now that the guemes in $S$ constit.nte a token passing ring with $|S|$ stations satisfying conditions (A1)-(A5) of Section 2 , whense operation is independent of the interarrival 
processes in the persistent culenes. The total inelinge switchover time $\bar{n}_{0}$ to this ring is equal to

$$
\bar{u}_{0}=u_{0}+\sum_{i \in l} \ell_{i} \cdot \bullet_{i}
$$

Let the quene lengths in such a system le denoted as $\left\{\overline{\mathrm{N}}_{\mathcal{S}}^{\prime \prime}(i)\right\}_{i \in \mathcal{S}}$. Clearly, $\overline{\mathrm{N}}_{\mathcal{S}}^{n}$ is a Markov chain, and since $|\mathcal{S}| \leq M-1$ we can apply the induction lyypothesis. Hence, for $i \in \mathcal{S}, \overrightarrow{\mathrm{N}}_{\mathcal{S}}^{n}$ is ergodic if

$$
\lambda_{i}<\frac{\ell_{i}}{u_{0}+\sum_{i \in U} \ell_{i} s_{i}}\left(1-\sum_{i \in S} \mu_{i}\right) \quad i \in \mathcal{S} .
$$

Assume now that (13) holds, and consider a quene in $\mathcal{S}$, say queue 1 and let $C_{s}^{\prime \prime}(1)$ be the process of cycle lengtlis (surcessive visits to queue 1). The process $\left(\mathrm{N}_{\mathcal{S}}^{n}(1), C_{\mathcal{S}}^{\prime \prime}(1)\right)$ is a Markov chain, as easy to motice. Since $N_{s}^{\prime \prime}(1)$ is ergorlic, it is easy to see that also the process $\left\{\overline{\mathbf{N}}_{s}^{n}(1), C_{s}^{n-1}(1)\right\}_{n=2}^{\infty}$ is stationary and ergorlic (for dletails see [3]). Now we are in position to use use Lemma 5 which inplies that the process $\bar{N}_{i}^{\prime \prime}(1), i \in \mathcal{U}$ is stable provided that

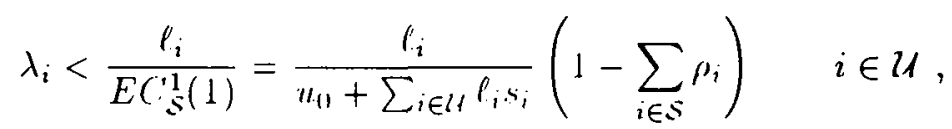

where the equality in (14) follows from the fact that hy Theorem 3 ,

$$
E\left(C_{s}^{1}(1)=\frac{\bar{u}_{01}}{1-\sum_{i \in S} \rho_{i}}=\frac{u_{01}+\sum_{j \in U_{1} \rho_{j} s_{j}}}{1-\sum_{j \in S} \rho_{j}}\right.
$$

Since the process $\bar{N}_{i}^{n}(1), \quad i \in S$ is stable by construction, it follows from (12) that the irreducible, aperiodic Markov chain $N^{\prime \prime}(1)$ is substable and therefore, ergodic. The fact that $\mathrm{N}^{n}(j)$ is ergodic for all $j \in \mathcal{M}$ follows from Theorem 3.

Putting everything together, from (13) and (14) we finally have that the Markov chain $\mathbf{N}^{n}(j)$ is ergorlic for every $j \in \mathcal{M}$ if

$$
\lambda_{i}<\frac{\ell_{i}}{u_{u}+\sum_{i \in U} \ell_{i} s_{i}}\left(1-\sum_{i \in S} \mu_{i}\right) \quad i \in \mathcal{M} .
$$

Since (16) holds for every partition $P=(\mathcal{S}, \mathcal{M})$ of the set $\mathcal{M}$ such that $\mathcal{S} \neq \mathcal{M}$, we conclude that the sufficient condition for stability of the system is

$$
R=\bigcup_{s \subset \mathcal{M}} R_{s}
$$

where

$$
\mathcal{R}_{S}=\left\{\lambda=\left(\lambda_{1}, \ldots, \lambda_{M}\right): \text { condition }(16) \text { holds }\right\} .
$$


Finally, to complete the proof wo need to show thlat

$$
\bigcup_{S \subset \mathcal{M}} \mathcal{R}_{S}=\left\{\lambda=\left(\lambda_{1}, \ldots, \lambda_{M}\right): \lambda_{i}<\frac{\ell_{i}}{u_{0}}\left(1-\sum_{i=1}^{M} \rho_{i}\right) i \in \mathcal{M}\right\} .
$$

This requires only algebraic: minuipulationss which are almost irlentical to the ones in [3], therefore we omit them liere. The interesting realer shonkl be able to reproduce this algebra.

Theorem 6 can be extended to innply the stalbility of the process $\overline{\mathrm{N}}(t)=\left(\tilde{N}_{1}(t), \cdots, \bar{N}_{M}(t)\right)$, where $\tilde{N}_{i}(t)$ is the quene length at. quene $i$ at. time $t$. We just only state the following.

Corollary 7. The proresss $\tilde{N}(t)$ is stulule if (11) holds.

Finally, we show in the next theorem that the conditions of Theorem (i are also necessary for the ergodicity of the Markov chain $N^{n}(i), i \in \mathcal{M}$. This will establish necessary condition for stability of the $\tau$-limited token passing ring, and therefore it completes the proof of Proposition 1.

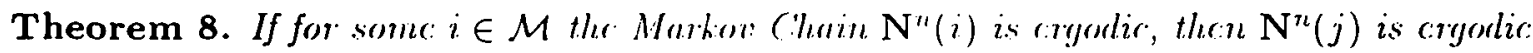
for every $i \in \mathcal{M}$. Morcow: $r, \sum_{i=1}^{M} \rho_{i}<1$, and

$$
\lambda_{j}<\frac{l_{1}}{u_{11}}\left(1-m_{11}\right), \quad j \in \mathcal{M} \text {. }
$$

Proof. The first assertion follows from Theorem 3 and the remark following that theorem. All cycles in the following will refer to cluent 1. For simplicity of notation we omit the queue index from the various variables. Let us define.

$C^{n}$ : length of the with cycle.

$C^{n}(r)$ : length of the nth cycle during which r customess from quene 1 were served.

$M^{n}(r)$ : number of cycles in regeneration cyclen $R^{n}$, (see proof of Theorem 3 for the definition of $R^{n}$ ) during which $r$ constomers were served. (learly,

$$
R=\sum_{r=0}^{\infty} M(r)
$$

where $M(r)=M^{1}(r)$ and $R=R^{1}$. 
Since (by the ergorlicity of the chain $\left.N^{\prime \prime}(1)\right) E R<\infty$, we have the following formulas for the long run averages:

- average length of a cycle during which $r$ custoners were serverl,

$$
E C(r)=\lim _{n \rightarrow \infty} \frac{\sum_{k=1}^{\prime \prime} C^{k}(r)}{n}=\frac{E\left(\sum_{k=1}^{M(r)} C^{k}(r)\right)}{E M(r)}
$$

- probability (proportion) of cycles cluring which $r$ customers were transmitterl,

$$
P(r)=\lim _{n \rightarrow \infty} \frac{\sum_{k=1}^{n} M^{k}(r)}{n}=\frac{E M(r)}{E R} .
$$

Consider now the following system.

System S. Upon arrival of the token to clueue 1, the number of customers (from quene 1) that will be served in the next cycle enters system $S$. These customers stay in $S$ until the token visits queue 1 for the next time, at which time all customers clepart.

Clearly, the number of customers that enter system $S$ in the $n$th cycle is $L_{1}^{n}$. Let $A_{S}^{t}$ be the number of customers that arrived in system $S$ by time $t$. Recall the definition of the renewal process $\tilde{C}^{\prime n}$ in the paragraph before Corollary 7. $A_{S}^{t}$ is regenerative with respect to $\tilde{C}^{n}$, and the ergodicity of $\mathrm{N}^{n}(1)$ implies by Theorem 3 that $E \tilde{C}^{\prime n}<\infty$. Hence we have that

$$
\lambda_{s}=\lim _{y \rightarrow \infty} \frac{A_{S}^{t}}{t}=\frac{\sum_{k=1}^{R} L_{1}^{k}}{\sum_{k=1}^{R} C^{k}}=\lambda_{1},
$$

where the last equality follows from (4). Similarly, taking into account that $E\left(\sum_{r=1}^{\infty} r M(r)\right)=$ $E\left(\sum_{k=1}^{R} L_{1}^{k}\right)<\infty$, we have the following formulas for the long-run average quene size, $E N_{S}$, and long-run average waiting time, $E W_{s}$, in system $\mathrm{S}$.

$$
\begin{aligned}
& E N_{S}=\frac{E\left(\sum_{r=1}^{\infty} r \sum_{k=1}^{M(r)}\left({ }^{k}(r)\right)\right.}{E\left(\sum_{k=1}^{F} C^{k}\right)}=\frac{\sum_{r=1}^{\infty} r E\left(\sum_{k=1}^{M(r)} C^{k k}(r)\right)}{E C, E R} \\
& E W_{S}=\frac{E\left(\sum_{r=1}^{\infty} r \sum_{k=1}^{M(r)} C^{k}(r)\right)}{E\left(\sum_{r=1}^{\infty} r M(r)\right)}=\frac{\sum_{r=1}^{\infty} r E\left(\sum_{k=1}^{M(r)} C^{k k}(r)\right)}{\sum_{r=1}^{\infty} r E(M(r))}
\end{aligned}
$$

Using (21), (22), we derive from (24), (2.5),

$$
\begin{aligned}
& E N_{S}=\frac{\sum_{r=1}^{\infty} r F^{\prime}(r) E(!(r)}{E(!}, \\
& E W_{S}=\frac{\sum_{r=1}^{\infty} r P^{\prime}(r) E(!(r)}{\sum_{r=1}^{\infty} r P^{\prime}(r)} .
\end{aligned}
$$


As in Theorem 1, let $L_{1}$ be a random varialle distributed as the steady state distribution of the process $\left\{L_{1}^{n}\right\}_{n=1}^{\infty}$. Then $\sum_{r=1}^{\infty} r I^{\prime}(r)=E L_{1}$. Since no more than $\bar{L}_{1}^{n}$ customers from quene $i$ are served during the $n$th cycle, it is easy to see that $L_{1} \leq_{s t} \tilde{L}_{1}^{1}$. If $E L_{1}=E \bar{L}_{1}^{1}$, then the stochastic dominance relation implies that $P^{\prime}\left(L_{1}=0\right)=P\left(L_{1}^{1}=0\right)=0$ and from (22) it follows that $E M(0)=0$. But then. $P^{\prime}\left(L^{n} \geq 1, \quad n=1,2 \cdots\right)=1$ and since $L^{n} \geq 1$ if and only if $N^{\prime \prime}(1) \geq 1$, we laves that $P^{\prime}\left(N^{\prime \prime}(1)=0, n=1,2 \cdots\right)=0$ which contradict the ergodicity of the chain $\mathrm{N}^{\prime \prime}(1)$. Therefore, $\sum_{r=1}^{\infty} r P^{\prime}(r)<E \bar{L}_{1}^{1}=\ell_{1}$ and

$$
E W_{S}>\frac{\sum_{r=1}^{\infty} r P(r) E C(r)}{\ell_{1}}
$$

Using Little's law (cf. [13]), (23) (26) antrl (2r) we have

$$
E N_{S}=\lambda_{S} E W_{S}>\lambda_{1} \frac{E N_{S} E C !}{\ell_{1}}
$$

and therefore,

$$
\lambda_{1} E C:<\ell_{1}
$$

and this proves Theorem 8 , and completes the proof of Proposition 1.

\section{References}

[1] S. Asmussen, Applicd Probulbility and Qurur.s, John Wiley\&.Sons, Chichester 1987.

[2] Chang C., Thomas .J., and Kiang S., ( $)_{11}$ the Stalility of $\mathrm{O}_{\mathrm{p}}$ en Networks: an Unified Approach by Stochastic Iominance, IBM R.: 18343, 1992.

[3] L. Georgiadis and W. Szprantowski, Stalbility of Token Passing Rings, Queucing Systems, $11(1992)$ pl. 7-333.

[4] O. Ibe and X. Cheng, Stability Comditions for Multiquene Systems with Cycle Service, IEEE Trans. Automutir: Comtrol, 333 (1958) 102-103.

[5] P. Kuehn, Multipnene Systenus with Nonexhanstive Cycle Service, The bcll Systcm Technical Journul, 58 (1979) (i71-(i9)s.

[6] K.K. Lenng and M. Eisenlerg, A Single-Sierver Quene with Vacations and Ciated TimeLimited Service, IEEE Trans. ('ommunu., 38 (1990) 1454-1462.

[7] K.K. Lenng and M. Eisenlerg, A Single-Sesver Qnene with Vacations and Non-Gated Time-Limited Service, Porf. Enoluntion .J., 12 (1991) 115-125.

[8] H. Levy, M. Sidli and (). Boxma, Dominance Relations in Polling Systems, Quencing Systcrns, (i (1990) 155-172.

[9] R. Loynes, The stability of a quene with non-independent inter-arival and service times, Prow: C'amblo. Pluiloss. Sior., sis (1!)(i2), 4!97-520). 
[10] V.A. Malyshev and M.V Mensikov. Ergodicity, continuity and analyticity of comntable Markov chains, Tranus. Moscoo Malls. Sior. (1!)s1), 1-1s.

[11] S. Meyn and D. Down, Stability of (ieneralized Jackson Networks. Ann. Appl. Prolubl, to appear.

[12] S. Meyn and R.L. Tweedle. (Giteria for Stability of Markovian Processes I: Discrete Time Chains, Adr. Appl. Prob., 24, 542-574, 1992.

[13] S. Stidham, Jr., A last worl on $L=\lambda W$. Oper. Rrs., 22 (1974), plp.417-421.

[14] W. Szpankowski, Towards computalule stability criteria for some multiclimensional stochastic processes, in Stochastic: Anolysis of Computcr and Communnication Siystcms (ed. H. Takagi), Elsevier Science Publications B. V. (North-Holland), (1990), p1. 131172.

[15] W. Szpankowski, Stability (ondlitions for Some Distributed Systems: Buffered Random Access Systems. Adu, Appl. Probmb. to appear.

[16] H. Takagi, Anulyysis of Follin! Siystcmus. The MIT Press, Cambridge, Mass. 1988.

[17] H. Takagi, Queneing Analysis of Polling Moclels, AC'M Computing Siurveys, 20 (1988) 5-28.

[18] R. L. Tweedie, (Giteria for classifying genemal Markov chains, Adv. Appl. Prob., 8 (1976), $737-771$.

[19] J. Walrand, An Introlurtion to Qumring Nrtumks, Prentice Hall, New Jersey 1988.

[20] K. Watson, Performance Evaluation of Cyclic Service Strategies - A Survey. Proc. PERFORMANCE'sh, E. (ieloulue Eal. (Paris 1!)44). 
Unité de Recherche INRIA Rocquencourt

Domaine de Voluceau - Rocquencourt - B.P. 105 - 78153 LE CHESNAY Cedex (France)

Unite de Recherche INRIA Lorraine Technopôle de Naney-Brabois - Campus Scientifique 615. rue Ju Jardin Botanique - B.P. 10! - 54602 VILLERS LES NANCY Cedex (France) Lnité de Recherche INRJA Rennes IRISA. Campus Universituire de Beaulieu 35042 RENNES Cedex (France) Unite de Recherche INRIA Rhöne-Alpes 46. avenue Félix Viallet - 38031 GRENOBLE Cedex (France)
Unite de Recherche INRIA Sophia Antipolis 20(44. route des Lucioles - B.P. $93-06902$ SOPHIA A.NTIPOLIS Cedex (France)

EDITEUR

INRIA - Domaine de Voluceau - Rocquencourt - B.P. 105 - 7815.3 LE CHESNAY Cedex (France)

ISSN $0249-6399$

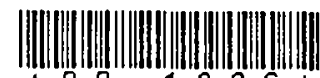

